\title{
Synthesis of formamide and isocyanic acid after ion irradiation of frozen gas mixtures
}

\author{
Z. Kaňuchováa ${ }^{1,3}$, R. G. Urso ${ }^{2,3}$, G. A. Baratta ${ }^{3}$, J. R. Brucato ${ }^{4}$, M. E. Palumbo ${ }^{3}$, and G. Strazzulla ${ }^{3}$ \\ 1 Astronomical Institute of Slovak Academy of Sciences, 05960 Tatranská Lomnica, Slovakia \\ e-mail: gianni@oact.inaf.it \\ 2 Dipartimento di Scienze Chimiche - Università degli Studi di Catania, Viale A. Doria 6, 95124 Catania, Italy \\ 3 INAF-Osservatorio Astrofisico di Catania, via Santa Sofia 78, 95123 Catania, Italy \\ ${ }^{4}$ INAF-Osservatorio Astrofisico di Arcetri, Largo Enrico Fermi 5, 50125 Firenze, Italy
}

Received 7 August 2015 / Accepted 5 November 2015

\section{ABSTRACT}

\begin{abstract}
Context. Formamide $\left(\mathrm{NH}_{2} \mathrm{HCO}\right)$ and isocyanic acid $(\mathrm{HNCO})$ have been observed as gaseous species in several astronomical environments such as cometary comae and pre- and proto-stellar objects. A debate is open on the formation route of those molecules, in particular if they are formed by chemical reactions in the gas phase and/or on grains. In this latter case it is relevant to understand if the formation occurs through surface reactions or is induced by energetic processing.

Aims. We present arguments that support the formation of formamide in the solid phase by cosmic-ion-induced energetic processing of ices present as mantles of interstellar grains and on comets. Formamides, along with other molecules, are expelled in the gas phase when the physical parameters are appropriate to induce the desorption of ices.

Methods. We have performed several laboratory experiments in which ice mixtures $\left(\mathrm{H}_{2} \mathrm{O}: \mathrm{CH}_{4}: \mathrm{N}_{2}, \mathrm{H}_{2} \mathrm{O}: \mathrm{CH}_{4}: \mathrm{NH}_{3}\right.$, and $\left.\mathrm{CH}_{3} \mathrm{OH}: \mathrm{N}_{2}\right)$ were bombarded with energetic $(30-200 \mathrm{keV})$ ions $\left(\mathrm{H}^{+}\right.$or $\left.\mathrm{He}^{+}\right)$. FTIR spectroscopy was performed before, during, and after ion bombardment. In particular, the formation of $\mathrm{HNCO}$ and $\mathrm{NH}_{2} \mathrm{HCO}$ was measured quantiatively.

Results. Energetic processing of ice can quantitatively reproduce the amount of $\mathrm{NH}_{2} \mathrm{HCO}$ observed in cometary comae and in many circumstellar regions. HNCO is also formed, but additional formation mechanisms are requested to quantitatively account for the astronomical observations.

Conclusions. We suggest that energetic processing of ices in the pre- and proto-stellar regions and in comets is the main mechanism to produce formamide, which, once it is released in the gas phase because of desorption of ices, is observed in the gas phase in these astrophysical environments.
\end{abstract}

Key words. astrochemistry - methods: laboratory: molecular - ISM: molecules - comets: general

\section{Introduction}

It is commonly thought that energetic processing of interstellar and planetary ices plays a relevant role in astrochemistry and astrobiology. There is also wide consensus that many complex molecules possibly formed in ice mantles on interstellar grains are not observable in the solid phase by IR spectroscopy (Allodi et al. 2013). It is also believed that the desorption of grain mantle species into the gas phase after warming by a proto-star or sputtering by energetic cosmic ions, for example, gives an important contribution to the gas phase composition (Modica \& Palumbo 2010; Palumbo et al. 2008). In this paper we present the formation of formamide $\left(\mathrm{NH}_{2} \mathrm{HCO}\right)$ and isocyanic acid $(\mathrm{HNCO})$ after ion irradiation of ice mixtures $\left(\mathrm{H}_{2} \mathrm{O}: \mathrm{CH}_{4}: \mathrm{N}_{2}, \mathrm{H}_{2} \mathrm{O}: \mathrm{CH}_{4}: \mathrm{NH}_{3}\right.$ and $\mathrm{CH}_{3} \mathrm{OH}: \mathrm{N}_{2}$ ).

Formamide is of considerable interest in prebiotic chemistry. Formamide and isocyanic acid have been observed as gaseous species in several astronomical environments such as cometary comae (e.g. Bockelée-Morvan et al. 2000; Biver et al. 2014); a new and extended set of recent gas phase observations of several high-, intermediate-, and low-mass pre- and proto-stellar objects has reinforced the interest in these molecules (Mendoza et al. 2014; López-Sepulcre et al. 2015). Formamide has also been tentatively identified in the solid phase in ISO-SWS infrared spectra (Schutte et al. 1999; Raunier et al. 2004). As a result of observations, some authors (Mendoza et al. 2014; López-Sepulcre et al. 2015) concluded that these two species are chemically related in star-forming regions and that at least formamide has to be produced in the solid phase and then released in the gas phase by thermal annealing. We note, however, that this view has recently been challenged by Barone et al. (2015), who have shown on the basis of theoretical calculations that formamide can be formed in the gas phase provided that its precursors, $\mathrm{NH}_{2}$ and $\mathrm{H}_{2} \mathrm{CO}$, are available.

This scenario stimulates the experimental studies of solid phase chemistry and in particular of the two main mechanisms that drive the chemical evolution of icy mantles in the interstellar medium (ISM), namely surface chemistry and energetic processing. Recent experiments of hydrogenation of isocyanic acid exclude the possibility that noteworthy quantities of formamide can be formed (Noble et al. 2015).

On the other hand, previous studies have shown that processing of various ice mixtures by UV photons (Bernstein et al. 1995; Henderson \& Gudipati 2015), energetic electrons (Jones et al. 2011; Bergantini et al. 2014; Henderson \& Gudipati 2015), and ions (Gerakines et al. 2004; Demyk et al. 1998) produce both formamide and isocyanic acid. The experimental results obtained so far are discussed in some detail in Sect. 2.1.

Here we extend these results and present a detailed quantitative measurement of the amount of synthesized formamide 
Table 1. Peak positions, vibration modes, molecule assignments, and band strengths.

\begin{tabular}{lcllcl}
\hline \hline \multicolumn{2}{l}{$\begin{array}{l}\text { Peak position } \\
\mathrm{cm}^{-1}\end{array}$} & $\mu \mathrm{m}$ & Vibration & Assign. & $\begin{array}{c}\text { Band strengths } \\
\left(\times 10^{-17} \mathrm{~cm} \mathrm{~mol}^{-1}\right)\end{array}$ \\
\hline 1388 & 7.2 & $v_{6}$ in plane $\mathrm{CH}$ sc. & $\mathrm{NH}_{2} \mathrm{HCO}$ & 0.68 & Brucato et al. (2006) \\
2260 & 4.42 & $\mathrm{~N}=\mathrm{C}=\mathrm{O}$ stretch & $\mathrm{HNCO}$ & 7.2 & van Broekhuizen et al. (2004) \\
\hline
\end{tabular}

and isocyanic acid after ion bombardment of ice mixtures $\left(\mathrm{H}_{2} \mathrm{O}: \mathrm{CH}_{4}: \mathrm{N}_{2}, \mathrm{H}_{2} \mathrm{O}: \mathrm{CH}_{4}: \mathrm{NH}_{3}\right.$, and $\left.\mathrm{CH}_{3} \mathrm{OH}: \mathrm{N}_{2}\right)$. In addition, some experiments performed on pure frozen formamide that have been published (Brucato et al. 2006) are re-analysed here. The results are discussed in the light of their astrophysical relevance, and we demonstrate that energetic processing of ice can quantitatively reproduce the amount of $\mathrm{NH}_{2} \mathrm{HCO}$ observed in cometary comae and in many circumstellar regions. HNCO is also formed, but additional formation mechanisms are required to quantitatively account for the astronomical observations.

\section{Energetic processing}

\subsection{Previous experiments}

Because it is generally agreed that hydrogenation reactions dominate the grain surface chemistry by saturating the molecules present in ice mantles, it seems obvious to assume that formamide is formed by hydrogenation of HNCO. Surprisingly, however, recent experiments have demonstrated that the hydrogenation of HNCO does not produce detectable amounts of formamide (Noble et al. 2015).

Several studies of energetic processing, with photons, electrons, and ions of different icy mixtures, have shown the formation of formamide and isocyanic acid. Bernstein et al. (1995) demonstrated the formation of formamide, acetamide, ethanol, and nitriles during the warming up of photolyzed samples made of ice mixtures containing $\mathrm{H}_{2} \mathrm{O}, \mathrm{CH}_{3} \mathrm{OH}, \mathrm{CO}$, and $\mathrm{NH}_{3}$. Henderson \& Gudipati (2015) have recently detected formamide produced by UV Lyman-alpha photons and energetic electrons by two-step laser ablation and ionization mass spectrometry. A detailed mechanistical study of the production of formamide after energetic electron bombardment of several $\mathrm{CO}: \mathrm{NH}_{3}$ ice mixtures has been presented by Jones et al. (2011). It has also been suggested that formamide is one of the products of ion irradiation and photolysis of $\mathrm{H}_{2} \mathrm{O}: \mathrm{HCN}$ (Gerakines et al. 2004) or $\mathrm{CO}: \mathrm{NH}_{3}$ (Demyk et al. 1998) at low temperature $(18 \mathrm{~K})$. In addition, samples of pure formamide ice were irradiated with $200 \mathrm{keV}$ protons (Brucato et al. 2006), and new molecules were synthesized, among which $\mathrm{CO}, \mathrm{CO}_{2}, \mathrm{~N}_{2} \mathrm{O}, \mathrm{HNCO}, \mathrm{NH}_{4}^{+}$ and $\mathrm{OCN}^{-}$.

This paper adds new experimental data on energetic processing of ice mixtures, and it is also the first to furnish a quantitative measurement of the molecular number ratio $\mathrm{HNCO} / \mathrm{NH}_{2} \mathrm{HCO}$ obtained from experiments that can be usefully compared with astronomical observations.

\subsection{Experimental apparatus}

Gaseous mixtures $\left(\mathrm{H}_{2} \mathrm{O}: \mathrm{CH}_{4}: \mathrm{N}_{2}\right.$, and $\mathrm{H}_{2} \mathrm{O}: \mathrm{CH}_{4}: \mathrm{NH}_{3}$ and $\mathrm{CH}_{3} \mathrm{OH}: \mathrm{N}_{2}$ ) were prepared in a vacuum chamber and were admitted through a needle valve into the scattering chamber $\left(P<10^{-7}\right.$ mbar $)$, where they accreted onto a cold $(10-20 \mathrm{~K})$ silicon or $\mathrm{KBr}$ substrate. Infrared transmittance spectra (resolution of $1 \mathrm{~cm}^{-1}$ ) were obtained before and after several steps of ion bombardment by FTIR spectrophotometers (Bruker Equinox 55 or Bruker Vertex 70). The substrates were in thermal contact with a closed-cycle helium cryostat (10-300 K).

An ion implanter (200kV; Danfysik 1080-200) generated energetic ions (up to $200 \mathrm{keV}$ per single ionization) that irradiated the sample on a spot greater than the area probed by the infrared beam (for more details on the experimental set-up see Strazzulla et al. 2001).

We here present experiments obtained by irradiating the samples with $30-200 \mathrm{keV} \mathrm{H}^{+}$or $\mathrm{He}^{+}$ions. The ion fluence in ions $\mathrm{cm}^{-2}$ was measured by a charge integrator and continuously monitored during irradiation. The substrate plane was placed at an angle of $45 \mathrm{deg}$ with respect to the IR beam and the ion beam so that spectra were taken in situ, even during irradiation, without tilting the sample.

The IR bands of a given molecule were used to measure the column density $N$ in units of molecules $\mathrm{cm}^{-2}$ through the formula

$N=\frac{\int \tau(v) \mathrm{d} v}{A}$,

where $\tau(v)$ is the optical depth and $A$ is the band strength $\left(\mathrm{cm}\right.$ molecule $\left.{ }^{-1}\right)$. The band strengths are listed in Table 1 together with band peak positions, assignments, and references.

Some of the experiments were performed by preparing frozen samples thinner than the penetration depth of the impinging ions that pass through the target and by obtaining the IR spectra at various steps of irradiation. Other experiments were made by irradiating the sample during its deposition, building up a larger thickness of processed material. In this way, the resulting spectrum better shows the weaker bands of the newly synthesized molecules. In the first case the energy deposited by incoming ions was obtained by calculating the stopping power (i.e. the amount of energy deposited per unit path length; $\mathrm{eV} \mathrm{cm} /$ molecule) of a given ion in a given target (SRIM code, Ziegler et al. 2008). By multiplying this number times the number of bombarding ions per square centimeter, we obtained the amount of energy released to the sample (dose) in $\mathrm{eV}$ permolecule. As usual, we express the dose in $\mathrm{eV} / 16 \mathrm{u}$, a convenient way to characterize chemical changes and to enable a comparison with other experiments with different samples.

Other samples are thicker than the penetration depth of the impinging ions that remain implanted in the target. In this case the dose was calculated by assuming that the energy of the incoming ion is uniformly absorbed by target molecule along the penetration depth of the ion.

For a simultaneous deposition of ices and irradiation, the energy released to the target molecules is estimated from the measurement of the ion flux (ions $\mathrm{cm}^{-2} \mathrm{~s}^{-1}$ ), the energy of impinging ions $(\mathrm{eV})$, and the accretion rate of the frozen film (molecules $\mathrm{cm}^{-2} \mathrm{~s}^{-1}$ ). In all of the experiments we used low current densities $\left(0.001-0.1 \mu \mathrm{A} \mathrm{cm}^{-2}\right)$ to avoid macroscopic heating of the target. 


\subsection{Results}

\subsubsection{Formamide}

Frozen layers of pure formamide were deposited at low temperature $(20 \mathrm{~K})$ and irradiated with $200 \mathrm{keV} \mathrm{H}^{+}$ions. In Fig. 1 (top panel) we show IR transmittance spectra $\left(2400-1250 \mathrm{~cm}^{-1}\right.$ $(4.17-8.0 \mu \mathrm{m}))$, in optical depth scale, of a deposited layer of formamide before and after irradiation at a dose of $12 \mathrm{eV} / 16 \mathrm{u}$. The thickness of the deposited layer was about $4900 \AA$, that is, less than the penetration depth of $200 \mathrm{keV} \mathrm{H}^{+}$ions (about $2 \mu \mathrm{m}$ ) in formamide, as calculated using the SRIM software (Ziegler et al. 2008).

The spectrum of formamide in the considered spectral range exhibits four bands at $1708,1631,1388$, and $1328 \mathrm{~cm}^{-1}$ before radiation (for more detail see Brucato et al. 2006). The decrease of the intensity of these bands and the appearance of new bands is evident from the figure and testifies to the formation of molecular species as indicated in the labels of Fig. 1 (top panel).

In the spectral range considered in Fig. 1, CO and $\mathrm{CO}_{2}$ absorption bands are observed at 2140 and $2342 \mathrm{~cm}^{-1}$, respectively. The bands observed at $2260 \mathrm{~cm}^{-1}$ and $2240 \mathrm{~cm}^{-1}$ are assigned to isocyanic acid (HNCO) and nitrous oxide $\left(\mathrm{N}_{2} \mathrm{O}\right)$, respectively. The weak band at $2083 \mathrm{~cm}^{-1}$ is due to the $\mathrm{CN}$ stretching mode in $\mathrm{HCN}$. The presence of the $\mathrm{SiH}$ stretching band observed at $1997 \mathrm{~cm}^{-1}$ is owing to the fraction of protons that entirely cross the ice sample and are implanted into the silicon substrate. Another intense band, located at $2165 \mathrm{~cm}^{-1}$, is commonly assigned to the cyanate anion $\mathrm{OCN}^{-}$ (Grim \& Greenberg 1987; Hudson et al. 2001; van Broekhuizen et al. 2004). Evidence that the ammonium cyanate complex $\mathrm{NH}_{4}^{+} \mathrm{OCN}^{-}$is formed is given by the band at $1478 \mathrm{~cm}^{-1}$, which is ascribed to $\mathrm{NH}_{4}^{+}$(Raunier et al. 2004).

Figure 2 gives the column density ratio of $\mathrm{HNCO} /$ formamide as a function of irradiation dose. It was obtained from the bands at $2260 \mathrm{~cm}^{-1}$ (HNCO) and $1388 \mathrm{~cm}^{-1}\left(\mathrm{NH}_{2} \mathrm{HCO}\right)$ using the band strength values reported in Table 1 . The HNCO band area was obtained by fitting the feature in the spectral range between $2270-2220 \mathrm{~cm}^{-1}$ with two Gaussian curves peaked at $2260 \mathrm{~cm}^{-1}$ and $2240 \mathrm{~cm}^{-1}$ that are assigned to HNCO and $\mathrm{N}_{2} \mathrm{O}$, respectively. The resulting error on the HNCO integrated optical depth is of the order of a few percent.

\subsection{2. $\mathrm{H}_{2} \mathrm{O}: \mathrm{CH}_{4}: \mathrm{N}_{2}$ mixtures}

Mixtures of $\mathrm{H}_{2} \mathrm{O}: \mathrm{CH}_{4}: \mathrm{N}_{2}(1: 1: 1)$ were deposited at $12 \mathrm{~K}$ and were at the same time irradiated with $30 \mathrm{keV} \mathrm{He}^{+}$beams. Experiments were conducted at low (about $10 \mathrm{eV} / 16 \mathrm{u}$ ) and high (about $300 \mathrm{eV} / 16 \mathrm{u}$ ) dose. In Fig. 1 (middle panel) we present the IR transmittance spectrum $\left(2400-1250 \mathrm{~cm}^{-1}(4.17-8.0 \mu \mathrm{m})\right)$ of such a mixture while it was irradiated by ions at a dose of about $300 \mathrm{eV} / 16 \mathrm{u}$. This spectrum clearly shows two features at 1388 and $1330 \mathrm{~cm}^{-1}$ that demonstrate the formation of formamide after ion bombardment. In the spectrum of the irradiated mixture we observe bands at about $1310 \mathrm{~cm}^{-1}$ and at about $1600 \mathrm{~cm}^{-1}$, which are due to the original frozen methane and water molecules, respectively. All of the other bands are formed after ion bombardment. In addition to formamide, we observe the appearance of the same bands observed after irradiation of formamide, although with different relative abundances: $\mathrm{CO}$ and $\mathrm{CO}_{2}$ at 2140 and $2342 \mathrm{~cm}^{-1}$, respectively; HNCO $\left(2260 \mathrm{~cm}^{-1}\right), \mathrm{N}_{2} \mathrm{O}\left(2240 \mathrm{~cm}^{-1}\right), \mathrm{OCN}^{-}\left(2165 \mathrm{~cm}^{-1}\right)$, $\mathrm{HCN}\left(2083 \mathrm{~cm}^{-1}\right)$, and $\mathrm{NH}_{4}^{+}\left(1478 \mathrm{~cm}^{-1}\right)$.
The column density ratio of $\mathrm{HNCO} /$ formamide was obtained by measuring their column density according to Eq. (1) using the area of the bands at $2260 \mathrm{~cm}^{-1}$ (HNCO) and at $1388 \mathrm{~cm}^{-1}$ (formamide) and their band strengths given in Table 1 . The results are reported in Fig. 2: the ratio at a low dose of irradiation values is about 3 and decreases to about 0.1 for high-dose irradiation. Moreover, in this case the HNCO band area was obtained by fitting the feature in the spectral range between $2270-2220 \mathrm{~cm}^{-1}$ with two Gaussian curves peaked at $2260 \mathrm{~cm}^{-1}$ and $2240 \mathrm{~cm}^{-1}$, which are assigned to $\mathrm{HNCO}$ and $\mathrm{N}_{2} \mathrm{O}$, respectively.

We performed additional experiments by changing the stoichiometry of the initial mixtures: $\mathrm{H}_{2} \mathrm{O}: \mathrm{CH}_{4}: \mathrm{N}_{2}(1: 2: 1) ;(1: 2: 10)$; and (1:2:100). These experiments were conducted with thick targets (i.e. larger than the penetration depth of the used ions) deposited at low $T(15-20 \mathrm{~K})$ and then irradiated with $30 \mathrm{keV}$ $\mathrm{He}^{+}$beams. The obtained column density ratios are plotted in Fig. 2. For these mixtures we also measured the total amount of deposited nitrogen atoms from which we obtained the fraction of nitrogen atoms that react to form $\mathrm{HNCO}$ and formamide. The results are plotted in Fig. 3 where the column density of HNCO and formamide formed after ion bombardment of the different mixtures is presented as the ratio to the total nitrogen atoms present before irradiation and plotted vs. irradiation dose $(\mathrm{eV} / 16 \mathrm{u})$.

Figure 2 shows that the ratio of $\mathrm{HNCO} /$ formamide decreases with the increase of nitrogen content (it is not measurable for the 1:2:100 mixture). Figure 3 clearly shows that both HNCO and formamide are formed less efficiently when the mixture is dominated by nitrogen; formamide is below the detection limit of our technique for the 1:2:100 mixture. The results are expected: it is reasonable that molecules that need $\mathrm{H}, \mathrm{C}, \mathrm{N}$ and $\mathrm{O}$ to be formed are less efficiently formed when one of the atoms dominates the mixture and the $\mathrm{C}$ - and $\mathrm{O}$-bearing species are dispersed in a matrix dominated by nitrogen.

\subsection{3. $\mathrm{H}_{2} \mathrm{O}: \mathrm{CH}_{4}: \mathrm{NH}_{3}$ mixture}

We prepared a thin frozen mixture of $\mathrm{H}_{2} \mathrm{O}: \mathrm{CH}_{4}: \mathrm{NH}_{3}(1: 1: 1)$ at $17 \mathrm{~K}$ and after deposition irradiated it with $200 \mathrm{keV} \mathrm{H}^{+}$beams. From a qualitative point of view we observe the formation of the same bands as for the previous mixtures, but with different relative intensities. A comparison of the spectra in Fig. 1 shows that substituting molecular nitrogen with ammonia causes a noteworthy increase in the relative amount of formamide with respect to that of isocyanic acid. This is made more clear in Fig. 2 where the ratio of the two species $\left(\mathrm{HNCO} / \mathrm{NH}_{2} \mathrm{HCO}\right)$ shows a behaviour completely different from the one of the mixtures with molecular nitrogen.

When ammonia is present, the amount of formed HNCO is much lower than that of formamide. Figure 3 shows that formamide is efficiently produced by incorporating up to about $15 \%$ of the initial nitrogen. HNCO is detected only at high dose, probably formed from species different from those deposited in the mixtures such as formamide itself.

\subsection{4. $\mathrm{CH}_{3} \mathrm{OH}: \mathrm{N}_{2}$ mixture}

We prepared a frozen mixture of $\mathrm{CH}_{3} \mathrm{OH}: \mathrm{N}_{2}(1: 1)$ as a thin film at $16 \mathrm{~K}$ and after deposition irradiated it with $200 \mathrm{keV}$ $\mathrm{H}^{+}$beams. We here also observe the formation of isocyanic acid and formamide, whose column density ratio is plotted in Fig. 2. The obtained values are clearly similar to those obtained with the molecular nitrogen containing ternary mixtures. This 

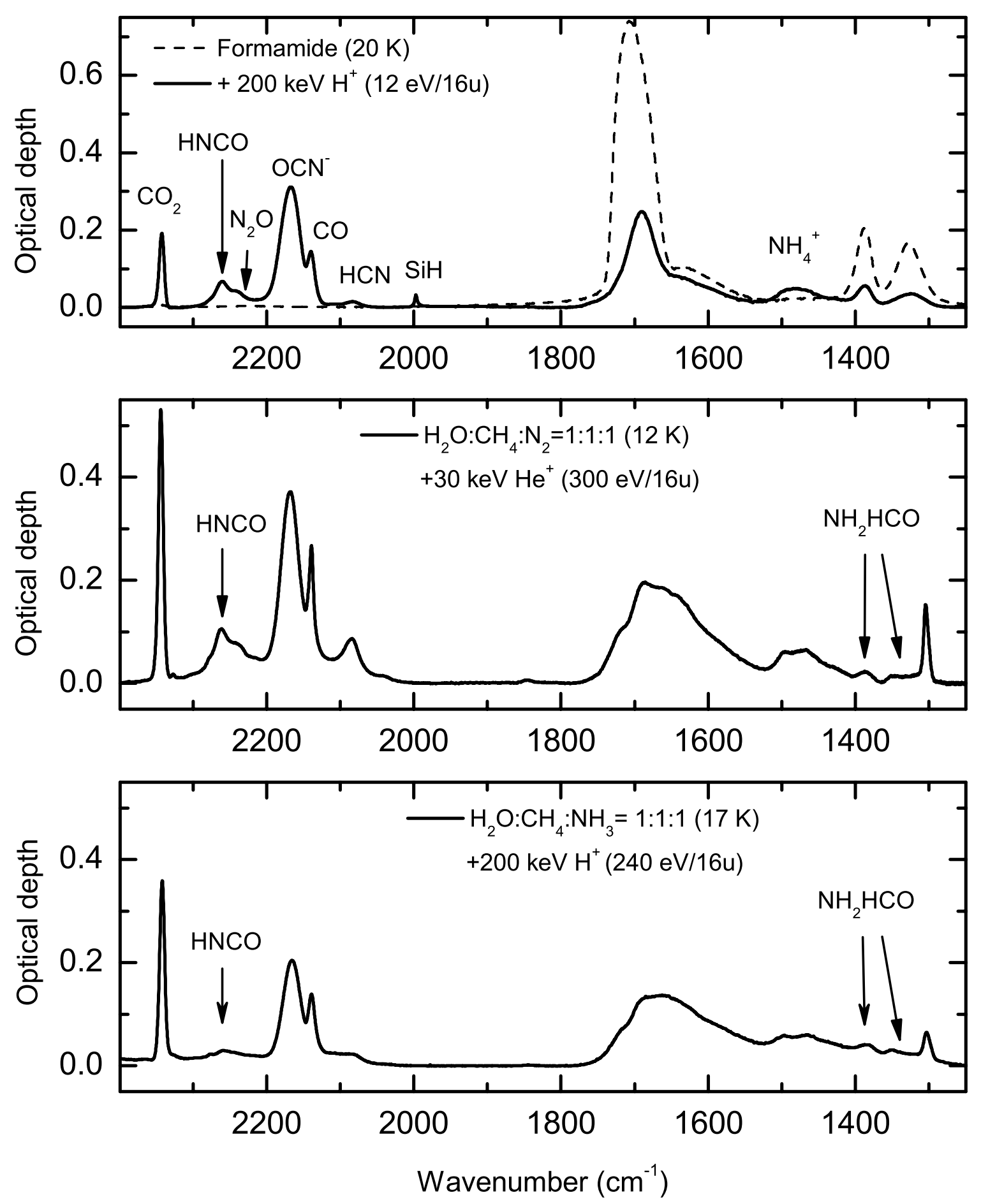

Fig. 1. IR transmittance spectra $\left(2400-1250 \mathrm{~cm}^{-1}(4.17-8.0 \mu \mathrm{m})\right)$, in optical depth scale. The top panel shows the spectrum of formamide (20 K) after irradiation at a dose of $12 \mathrm{eV} / 16 \mathrm{u}$ with $200 \mathrm{keV} \mathrm{H}^{+}$ions (full line). The main species formed after irradiation are labelled. The spectrum obtained before irradiation is also shown for comparison (dotted line). The middle panel shows the spectrum of a mixture $\mathrm{H}_{2} \mathrm{O}: \mathrm{CH}_{4}: \mathrm{N}_{2}(1: 1: 1)$ deposited at $12 \mathrm{~K}$ and while being irradiated by ions at a dose of about $300 \mathrm{eV} / 16 \mathrm{u}$. Absorption features assigned to $\mathrm{HNCO}$ and $\mathrm{NH}_{2} \mathrm{HCO}$ are labelled. The bottom panel shows the spectrum of a mixture $\mathrm{H}_{2} \mathrm{O}: \mathrm{CH}_{4}: \mathrm{NH}_{3}(1: 1: 1)$ as deposited at $17 \mathrm{~K}$ and after irradiation with $200 \mathrm{keV} \mathrm{H}^{+}$of about $240 \mathrm{eV} / 16 \mathrm{u}$. Absorption features assigned to $\mathrm{HNCO}$ and $\mathrm{NH}_{2} \mathrm{HCO}$ are labelled.

consistency is confirmed by the data reported in Fig. 3, which show a ratio of $\mathrm{HNCO} /($ initial nitrogen atoms) of about 6-7 per mill and a ratio of 2-3 per cent for formamide/(initial nitrogen atoms).

The quantitative results reported in Fig. 3 are consistent with previous experimental results. Bernstein et al. (1995) reported a quantity of about $1.8 \%$ (with respect to original nitrogen) of formamide in the residue formed after UV photolysis and thermal processing of methanol-containing interstellar ice analogues. Jones et al. (2011) found an abundance of formamide of about $1-2 \%$ after 60 min of electron bombardment of CO: $\mathrm{NH}_{3}$ mixtures at $12 \mathrm{~K}$. Gerakines et al. (2004) found an abundance of about $2 \%$ after $0.8 \mathrm{MeV}$ proton irradiation of $\mathrm{H}_{2} \mathrm{O}: \mathrm{HCN}$ and $\mathrm{H}_{2} \mathrm{O}: \mathrm{NH}_{3}: \mathrm{HCN}$ ice mixture at a dose of $10 \mathrm{eV} / \mathrm{mol}$. 
Z. Kaňuchová et al.: Formamide and isocyanic acid after ion irradiation of ices

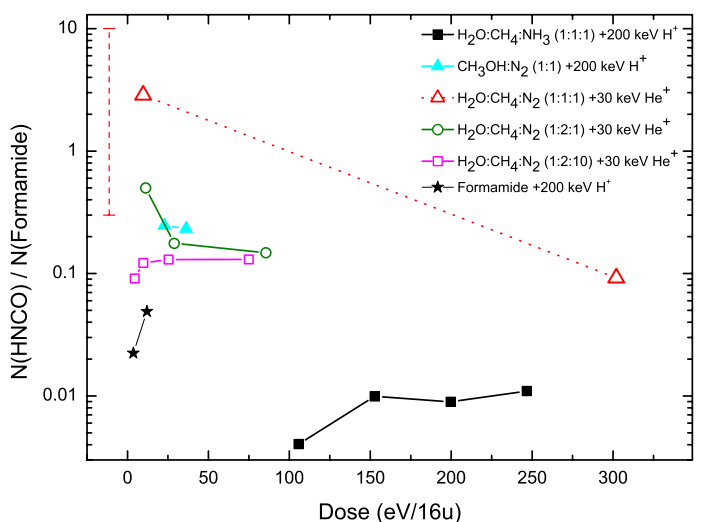

Fig. 2. Molecular number ratio $\mathrm{HNCO} /$ formamide obtained after ion bombardment of different frozen ice mixtures is plotted vs. irradiation dose $(\mathrm{eV} / 16 \mathrm{u})$. The dashed vertical line represents the range of values measured in astronomical sources.

\section{Discussion}

Water $\left(\mathrm{H}_{2} \mathrm{O}\right)$, carbon monoxide $(\mathrm{CO})$, carbon dioxide $\left(\mathrm{CO}_{2}\right)$, methanol $\left(\mathrm{CH}_{3} \mathrm{OH}\right)$, methane $\left(\mathrm{CH}_{4}\right)$, and ammonia $\left(\mathrm{NH}_{3}\right)$ are the most abundant molecular species detected in the interstellar medium in the solid phase (e.g. Gibb et al. 2004; Öberg et al. 2011). These species are also observed in cometary comae (e.g. Bockelée-Morvan et al. 2000). Molecular nitrogen, being a homonuclear molecule without any infrared active band, cannot be directly observed in icy grain mantles, but its presence is deduced from the depletion of gas-phase species (e.g. Caselli et al. 2002; Bergin et al. 2002). Recently, molecular nitrogen has been detected in comet 67P/Churyumov-Gerasimenko (Rubin et al. 2015). In the present study we have considered binary and ternary mixtures with $\mathrm{H}-\mathrm{O}-\mathrm{C}$ - and $\mathrm{N}$-bearing species (i.e. $\mathrm{H}_{2} \mathrm{O}$, $\mathrm{CH}_{3} \mathrm{OH}, \mathrm{CH}_{4}, \mathrm{NH}_{3}$ and $\mathrm{N}_{2}$ ). All these mixtures are reasonably representative of the composition of interstellar icy grain mantles and cometary ices. For the $\mathrm{H}_{2} \mathrm{O}: \mathrm{CH}_{4}: \mathrm{N}_{2}$ mixture we also considered different initial ratios to investigate how the relative abundance of a given species can affect the results.

\subsection{Pre- and proto-stellar regions}

Solid-phase formamide has been tentatively identified in ISO-SWS infrared spectra of the proto-stellar objects NGC 7538 IRS9 and W33A (Schutte et al. 1999; Raunier et al. 2004). The observations in the gaseous phase are more reliable. Its first detection was towards Sgr B2 (Rubin et al. 1971), and it was subsequently observed in molecular clouds and high-mass star-forming regions (Nummelin et al. 2000; Bisschop et al. 2007; Motiyenko et al. 2012). HNCO has not yet been observed in the solid phase, although it is believed to be present, formed by surface chemistry or energetic processing, and to be responsible for the formation of the abundant cyanate ion $\mathrm{OCN}^{-}$(e.g. Soifer et al. 1979; Demyk et al. 1998; Lowenthal et al. 2002; van Broekhuizen et al. 2005). It has been observed in the gas phase in numerous pre- and proto-stellar regions (Bisschop et al. 2007; Marcelino et al. 2009; Rodríguez-Fernández et al. 2010).

An extended set of recent observations of both formamide and isocyanic acid have renewed the interest in these molecules (Mendoza et al. 2014; López-Sepulcre et al. 2015). Mendoza et al. (2014) studied these molecules and observed their emission in the proto-stellar shock regions L1157-B1 and L1157-B2 with the IRAM 30 m radio-telescope. By comparing their results with those already available, they found a linear correlation between

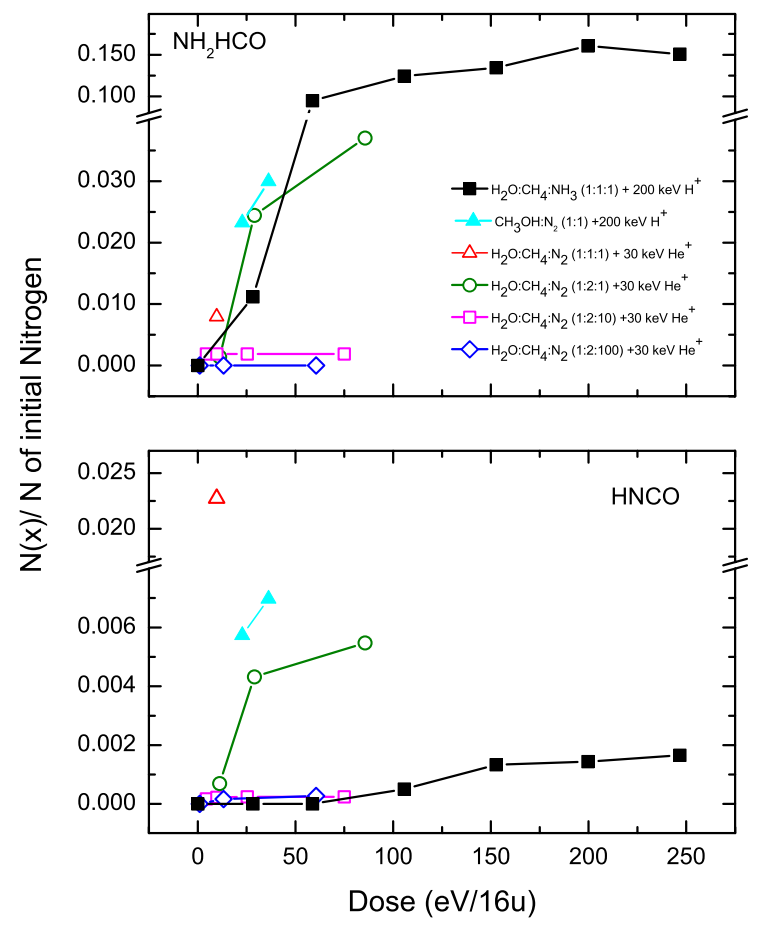

Fig. 3. Column density of HNCO and formamide formed after ion bombardment of different frozen ice mixtures shown as the ratio to the total nitrogen atoms present in the mixtures before irradiation and plotted vs. irradiation dose $(\mathrm{eV} / 16 \mathrm{u})$.

the abundances of the two molecules, with HNCO being about three times more abundant than $\mathrm{NH}_{2} \mathrm{HCO}$. This implies that the two species are chemically related. The authors suggested molecule formation on icy grain mantles. Further observations of ten low- and intermediate-mass pre- and proto-stellar objects allowed detecting $\mathrm{HNCO}$ in all of the sources and $\mathrm{NH}_{2} \mathrm{HCO}$ in only five of them (López-Sepulcre et al. 2015). An almost linear correlation between $\mathrm{HNCO}$ and $\mathrm{NH}_{2} \mathrm{HCO}$ abundances was confirmed. The fractional abundance (with respect to molecular hydrogen) of HNCO varies from source to source between $2 \times 10^{-10}$ and $10^{-7}$ and the fractional abundance of $\mathrm{NH}_{2} \mathrm{HCO}$ varies between $2 \times 10^{-11}$ and $10^{-8}$ (Mendoza et al. 2014).

When all of the available observations are considered in a graph where the ratio $\mathrm{HNCO} / \mathrm{NH}_{2} \mathrm{HCO}$ is plotted vs. the luminosity of the source (which spans over six orders of magnitude; see the bottom panel of Fig. 3 in López-Sepulcre et al. 2015), the objects are concentrated in two regions. The first contains objects with a very high ratio (i.e. no or very few formamide has been detected), the other region contains sources for which the two molecules are observed and the ratio spans between about 10 and 0.3. The sources without formamide detection are also the coldest and devoid of hot corinos. The authors concluded that while $\mathrm{HNCO}$ can be formed in the gas-phase during the cold stages of star formation, formamide forms mostly on the mantles of dust grains at low temperatures and is expelled into the gas phase when the temperature rises enough to sublimate the icy grain mantles.

Recently, Barone et al. (2015) have studied the reaction $\mathrm{NH}_{2}+\mathrm{H}_{2} \mathrm{CO} \rightarrow \mathrm{NH}_{2} \mathrm{HCO}+\mathrm{H}$ in theory and found that it can efficiently form formamide in the gas-phase in star-forming regions. They therefore concluded that there is no need to invoke grain-surface chemistry to explain the presence of formamide provided that its precursors $\mathrm{NH}_{2}$ and $\mathrm{H}_{2} \mathrm{CO}$ are available in the gas phase. 
Here we presented the results of laboratory experiments that show the formation of $\mathrm{HNCO}$ and $\mathrm{NH}_{2} \mathrm{HCO}$ after ion bombardment of astrophysical relevant ice mixtures. We have found that at low dose (about $10 \mathrm{eV} / 16 \mathrm{u}$; Fig. 3), the amount of $\mathrm{NH}_{2} \mathrm{HCO}$ formed is of the order of a few percent with respect to available nitrogen in the ice mixtures, while the amount of $\mathrm{HNCO}$ formed is about one order of magnitude less. The cosmic abundance of nitrogen, as reported by Asplund et al. (2009), is $6.8 \times 10^{-5}$. In their study of dark clouds, Maret et al. (2006) adopted an elemental nitrogen fractional abundance of $2 \times 10^{-5}$. If we take into account the value given by Maret et al. (2006), and if we assume that in dense cold proto-stellar regions most nitrogen is in the solid phase, then the amount of $\mathrm{NH}_{2} \mathrm{HCO}$ that can be formed in icy grain mantles can be as high as $10^{-7}$ with respect to hydrogen. Similarly, the amount of HNCO can be as high as $10^{-8}$ with respect to hydrogen. When the column density ratio $\mathrm{HNCO} / \mathrm{NH}_{2} \mathrm{HCO}$ is plotted versus dose (see Fig. 2), we note that at the dose values we investigated, the ratio obtained in laboratory is in most cases lower than the ratio observed in starforming regions (López-Sepulcre et al. 2015). These experimental results indicate that while the observed amount of formamide can be accounted for by cosmic-ray bombardment of icy grain mantles, other processes (such as gas-phase and grain-surface reactions) have to be taken into account to explain the observed gas-phase abundance of HNCO. This suggestion is based on the assumption that after desorption of ice grain mantles, molecules released to the gas phase do not suffer from further processing.

To estimate the time necessary to obtain in star-forming regions the effects observed in the laboratory, we considered the approximation of effective monoenergetic $1 \mathrm{MeV}$ protons (Mennella et al. 2003). Furthermore, our experimental results were obtained using $200 \mathrm{keV}$ protons or $30 \mathrm{keV} \mathrm{He}$ ions. To extrapolate the laboratory results to the interstellar medium conditions, we therefore assumed that they scale with the stopping power ( $\mathrm{S}$, energy loss per unit path length) of impinging ions (e.g. Palumbo 2006; Sicilia et al. 2012). Then the time is given by

$t_{\mathrm{ISM}}=\frac{S_{\text {lab }} \times \text { Fluence }_{\text {lab }}}{S_{\mathrm{ISM}} \times \Phi_{\mathrm{ISM}}}$,

where $S_{\text {lab }}$ and $S_{\text {ISM }}$ are the stopping power of $200 \mathrm{keV}$ protons (or $30 \mathrm{keV} \mathrm{He}^{+}$) and $1 \mathrm{MeV}$ protons as obtained from SRIM software (Ziegler et al. 2008), respectively. Fluence lab $_{\text {ab }}$ is the fluence (ions $\mathrm{cm}^{-2}$ ) as measured during the experiments, and $\Phi_{\text {ISM }}$ is the flux (ions $\mathrm{cm}^{-2} \mathrm{~s}^{-1}$ ) of $1 \mathrm{MeV}$ protons in starforming regions. This has to be regarded as an effective quantity: it represents the equivalent flux of $1 \mathrm{MeV}$ protons that gives rise to the ionization rate produced by the cosmic ray spectrum if $1 \mathrm{MeV}$ protons were the only source for ionization (Mennella et al. 2003). Table 2 gives the time required in star-forming regions calculated assuming different values of the ionization rate $\left(\zeta^{\mathrm{H}_{2}}\left(\mathrm{~s}^{-1}\right)\right)$, as reported by Mennella et al. (2003), Podio et al. (2014), and Woods et al. (2015). The ionization rate values we considered fall in the range predicted by models (e.g. Padovani et al. 2009, 2013).

The lifetime of pre- and proto-stellar phases have been estimated to be of the order of $10^{5}-10^{7}$ yr (Larson 2003; Caselli \& Ceccarelli 2012), thus the results presented in the table demonstrate that the dust has the chance of accumulating a dose of the order of a few eV/16u before the ices desorb. Thus we suggest that most of the formamide observed in these regions is formed by energetic processing of ices.
Table 2. Time scales (years) to accumulate a dose of 1,10 and $100 \mathrm{eV} / 16 \mathrm{u}$ by cosmic ion irradiation of icy mantles of dust grains in pre- and proto-stellar regions.

\begin{tabular}{lcccc}
\hline \hline Dose $(\mathrm{eV} / 16 \mathrm{u})$ & \multicolumn{4}{c}{ Timescale $(\mathrm{yr})$} \\
\hline & & \multicolumn{3}{c}{ ionization rate $\left(\mathrm{s}^{-1}\right)$} \\
& $1.3 \times 10^{-17}$ & $6 \times 10^{-17}$ & $3 \times 10^{-16}$ & $1.3 \times 10^{-15}$ \\
\hline 1 & $1.1 \times 10^{7}$ & $2.5 \times 10^{6}$ & $5.0 \times 10^{5}$ & $1.1 \times 10^{5}$ \\
10 & $1.1 \times 10^{8}$ & $2.5 \times 10^{7}$ & $5.0 \times 10^{6}$ & $1.1 \times 10^{6}$ \\
100 & $1.1 \times 10^{9}$ & $2.5 \times 10^{8}$ & $5.0 \times 10^{7}$ & $1.1 \times 10^{7}$ \\
\hline
\end{tabular}

Notes. The time scales have been estimated for different hydrogen ionization rates $\left(\zeta^{\mathrm{H}_{2}}\left(\mathrm{~s}^{-1}\right)\right)$ in the range measured in different regions.

\subsection{Comets}

Formamide and isocyanic acid have also been observed in the coma of some comets. $\mathrm{HNCO} / \mathrm{NH}_{2} \mathrm{HCO}$ has been measured for three comets: in the long-period C/1995 O1 HaleBopp (Bockelée-Morvan et al. 2000), and recently in comets C/2012 F6 (Lemmon) and C/2013 R1 (Lovejoy) (Biver et al. 2014). It is interesting to note that the molecular number ratios $\mathrm{HNCO} / \mathrm{NH}_{2} \mathrm{HCO}$ are 5 (in the coma of Hale-Bopp), 1.6 (Lemmon), and 1 (Lovejoy). These values are interestingly compatible with those measured in the pre- and proto-stellar regions of young stars. The existence of a link between the composition of the interstellar material and comets has been suggested for a long time (e.g. Greenberg 1982). The hypothesis is that when interstellar grains accrete onto planetesimals and small bodies, they preserve their composition, at least in part.

It is also known, however, that comets are continuously exposed to energetic processes such as cosmic ion and UV photon irradiation, which are able to alter the original cometary material and induce chemical and physical changes, including the formation of molecules that were not originally present. As an example applying experimental results to a specific molecule, it has been suggested that methyl formate is formed in comets after ion bombardment of methanol-bearing ices. It has been evaluated that such a process can account for about $12 \%$ of the methyl formate observed in comet Hale-Bopp (Modica et al. 2012). The chemical changes studied in the laboratory have also been used to predict the development of a refractory organic crust that inhibits a direct exposure of ices at the surface (Strazzulla et al. 1991), as presently observed for comet 67P/ChuryumovGerasimenko by the VIRTIS instrument on the Rosetta spacecraft (Capaccioni et al. 2015). At deeper layers, until about a depth of $5 \mathrm{~m}$, the nucleus is depleted in volatiles and would have a considerable fraction of organic molecules formed because of irradiation along with unrecombined radicals (Strazzulla \& Johnson 1991). Near the surface the dose deposited by cosmic ions is high (hundreds of $\mathrm{eV} / 16 \mathrm{u}$ ) and decreases with the depth. Doses as low as a few $\mathrm{eV} / 16 \mathrm{u}$ are accumulated at depth of the order of tens of meters. In addition to the external flux of cosmic ions, the decay of incorporated radioactive elements also produces chemical effects in comets. The radiation from radionuclides consists of the contribution of long-lived (e.g. ${ }^{129} \mathrm{I},{ }^{247} \mathrm{Cm}$, ${ }^{244} \mathrm{Pu}$ ) and shorted-lived (e.g. ${ }^{10} \mathrm{Be},{ }^{237} \mathrm{~Np}$, and $\left.{ }^{26} \mathrm{Al}\right)$ radionuclides. The absorbed dose that has been accumulated throughout the nucleus over the comet lifetime $\left(4.6 \times 10^{9} \mathrm{yr}\right)$ has been estimated to be about $2.3 \mathrm{eV} / 16 \mathrm{u}$, of which about $0.5 \mathrm{eV} / 16 \mathrm{u}$ are due to long-living nuclides and about $1.8 \mathrm{eV} / 16 \mathrm{u}$ to the shorter living ${ }^{26} \mathrm{Al}$ (Draganić \& Draganić 1984). In the first meter of depth this dose is much lower than that due to cosmic rays. Nevertheless, 
Table 3. Abundances (with respect to water) of relevant molecules measured for the three comets where HNCO and formamide have been observed.

\begin{tabular}{cllll}
\hline \hline \multirow{2}{*}{ Species } & \multicolumn{3}{c}{ Abundance $\left(\%\right.$ of $\left.\mathrm{H}_{2} \mathrm{O}\right)$} & References \\
& Hale-Bopp & Lemmon & Lovejoy & \\
\hline $\mathrm{CO}$ & 2.3 & 4.0 & 7.2 & Bockelée-Morvan et al. (2000), Biver et al. (2014) \\
$\mathrm{N}_{2}$ & 0.013 & 0.023 & 0.041 & estimated from Rubin et al. (2015) \\
$\mathrm{HNCO}$ & 0.1 & 0.025 & 0.021 & Bockelée-Morvan et al. (2000), Paganini et al. (2014a) \\
$\mathrm{NH}_{2} \mathrm{HCO}$ & 0.02 & 0.06 & 0.021 & Bockelée-Morvan et al. (2000), Biver et al. (2014) \\
$\mathrm{NH}_{3}$ & $1-1.8$ & $\leq 0.63$ & $\leq 1.86$ & Biver et al. (2014), Paganini et al. (2014a, b) \\
\hline $\mathrm{HNCO}^{\mathrm{N}}$ in $\mathrm{N}_{2}$ & 3.85 & 0.55 & 0.26 & \\
$\mathrm{NH}_{2} \mathrm{HCO} / \mathrm{N}_{\text {in }} \mathrm{N}_{2}$ & 0.77 & 1.3 & 0.26 & \\
& & & & \\
$\mathrm{HNCO} / \mathrm{NH}_{3}$ & $0.06-0.1$ & $\geq 0.04$ & $\geq 0.01$ & \\
$\mathrm{NH}_{2} \mathrm{HCO} / \mathrm{NH}_{3}$ & $0.01-0.02$ & $\geq 0.01$ & $\geq 0.01$ & \\
\hline
\end{tabular}

Notes. The ratios of $\mathrm{HNCO}$ and formamide with respect to nitrogen atoms in $\mathrm{N}_{2}$ or in $\mathrm{NH}_{3}$ are also reported.

it is important for the total amount of ionizing energy accumulated throughout the nucleus over the comet lifetime because its action is not limited to surface layers, but extends along the entire cometary nucleus that is uniformly irradiated. As demonstrated in this paper and in the literature quoted in Sect. 2.1, formamide and HNCO are formed by energetic processing of frozen mixtures containing simple $\mathrm{H}-, \mathrm{C}-, \mathrm{N}-$, and O-bearing molecules.

Therefore it makes sense to investigate whether energetic processing of comets can contribute to the formation of the two N-bearing molecules. In comet Hale-Bopp ammonia has been detected in the coma with an abundance of about $1-1.8 \%$ with respect to water (Bird et al. 1997; Crovisier \& BockeléeMorvan 1999). Molecular nitrogen is thought to have been the most abundant form of nitrogen in the proto-solar nebula, although it has only recently been observed in situ in comet 67P/Churyumov-Gerasimenko by the Rosetta Orbiter Spectrometer for Ion and Neutral Analysis mass spectrometer onboard the Rosetta spacecraft (Rubin et al. 2015). The molecular number ratio for $\mathrm{N}_{2} / \mathrm{CO}$ of $5.70 \pm 0.66 \times 10^{-3}$ implies a depletion of $25.4 \pm 8.9$ with respect to the available pre-solar nitrogen. In Table 3 we report the abundances of relevant molecules measured for the only three comets where HNCO and formamide have been observed. We have scaled the abundance of $\mathrm{N}_{2}$ from that of $\mathrm{CO}$ assuming the same ratio as measured for comet 67P/Churyumov-Gerasimenko by Rubin et al. (2015). In Table 3 we also report the ratios of $\mathrm{HNCO}$ and formamide with respect to nitrogen atoms in $\mathrm{N}_{2}$ or in $\mathrm{NH}_{3}$. This number can be compared with the results presented in Fig. 3, with the caution that in the figure the ratio refers to the nitrogen originally present, while from the astronomical observations it is not possible to determine to which extent the observed molecular abundances are pristine or modified by post-formation processing. With this in mind, we see, however, that in comets HNCO cannot be produced by energetic processing of the studied ices in significant amount. In addition, assuming that all of the pre-solar molecular nitrogen was incorporated into comets, the ratios in the table would be divided by a factor 25 , which is still higher than measured in the laboratory. We then conclude that either HNCO is incorporated in the comet directly from the gas in the solar nebula or it has to be produced by processing of ice mixtures different from those considered here. The case of formamide is different: assuming, conservatively, that the observed ammonia is the pristine one, the results shown in Fig. 3 indicate that the observed ratio of formamide/ammonia can be obtained after a dose of about a few eV/16u.

\subsection{Astrobiological relevance of formamide}

Among the different theories for the prebiotic chemistry, the extraterrestrial synthesis of organic compounds and their delivery to the Earth's surface is seen as one of more probable scenarios that occurred on the early Earth and that was responsible for the origin of life. A considerable amount of extraterrestrial material was and is continuously delivered to Earth (between $10^{7}$ and $10^{9} \mathrm{~kg} \mathrm{yr}^{-1}$ of organics in the first billion years; Chyba \& Sagan 1992).

Formamide $\left(\mathrm{NH}_{2} \mathrm{HCO}\right)$ is the simplest naturally occurring amide. This compound contains in its structure all the elements that are required for the synthesis of biomolecules, specifically, hydrogen, carbon, oxygen, and nitrogen, with the only exception of phosphorus and sulphur. Although formamide is easily formed by the reaction of hydrogen cyanide with water, we here suggest that it can be directly delivered to Earth by comets and cometary debris. It is a polar solvent like water with the characteristic that many species that are unstable in water with respect to hydrolysis are spontaneously synthesized in formamide. This includes nucleosides (from ribose borates and nucleobases), peptides (from amino acids), and even oligoribonucleotides. Thus its presence on the early Earth could have favoured a further chemical evolution.

An intense experimental analysis has in fact demonstrated that formamide is a very active molecule in the synthesis of fundamental compounds of biochemistry, such as nucleobases and carboxylic acids.

Thus, formamide chemistry is an extremely promising pathway to provide a plausible unitary frame for the origin of the genetic polymers and metabolic cycles in the extant forms of life (Saladino et al. 2012).

Acknowledgements. This work was supported by the Italian Ministero dell'Istruzione, Università e Ricerca through the grant Progetti Premiali 2012iALMA (CUP C52I13000140001). This work was also supported by COST Action TD 1308. The research of Z.K. was supported by VEGA - The Slovak Agency for Science, Grant No. 2/0032/14.

\section{References}

Allodi, M. A., Baragiola, R. A., Baratta, G. A., et al. 2013, Space Sci. Rev., 180, 101

Barone, V., Latouche, C., Skouteris, D., et al. 2015, MNRAS, 453, L31 Bergantini, A., Pilling, S., Nair, B. G., et al. 2014, A\&A, 570, A120 Bernstein, M. P., Sandford, S. A., Allamandola, L. J., et al. 1995, ApJ, 454, 327 Bird, M. K., Huchtmeier, W. K., Gensheimer, P., et al. 1997, A\&A, 325, L5 Bisschop, S. E., Jorgensen, J. K., van Dishoeck, E. F., et al. 2007, A\&A, 465, 913 
Biver, N., Bockelée-Morvan, D., Debout V., et al. 2014, A\&A, 566, L5 Bockelée-Morvan, D., Lis, D. C., Wink, J. E., et al. 2000, A\&A, 353, 1101 van Broekhuizen, F. A., Pontoppidan K. M., Fraser, H. J., \& van Dishoeck E. F. 2005, A\&A, 441, 249

van Broekhiuzen, F. A., Keane, J. V., \& Schutte, W. A. 2004, A\&A, 415, 425

Brucato, J. R., Baratta, G. A., \& Strazzulla, G. 2006, A\&A, 455, 395

Capaccioni, F., Corradini, A., Filacchione, G., et al. 2015, Science, 347, 0628

Caselli, P., \& Ceccarelli, C. 2012, A\&ARv, 20, 56

Chyba, C. F., \& Sagan, C. 1992, Nature, 255, 125

Crovisier, J., \& Bockelée-Morvan, D. 1999, Space Sci. Rev., 90, 19

Draganić, I. G., \& Draganić, Z. D. 1984, Adv. Space Res., 4, 115

Demyk, K., Dartois, E., d'Hendecourt, L., et al. 1998, A\&A, 339, 553

Fulvio, D., Sivaraman, B., Baratta, G. A., et al. 2009, Spectrochim. Acta Part A, 72,1007

Gerakines, P. A., Moore, M. H., \& Hudson, R. L. 2004, Icarus, 170, 202

Gibb, E. L., Whittet, D. C. B., Boogert, A. C. A., \& Tielens, A. G. G. M. 2004 , ApJSS, 151, 35

Greenberg, J. M., 1982, in Comets. (A83-13376 03-90) (Tucson, AZ: University of Arizona Press), 131

Grim, R. J. A., \& Greenberg, J. M. 1987, ApJ, 321, L91

Henderson, H. B., \& Gudipati, M. S. 2015, 800, 66

d'Hendecourt, L., \& Allamandola, J. L. 1986, A\&AS, 64, 453

Hudson, R. L., Moore, M. H., \& Gerakines, P. A. 2001, ApJ, 550, 1140

Jiang, G. J., Person, W. B., \& Brown, K. G. 1975, J. Chem. Phys., 62, 1201

Jones, B., Bennett, C. J., \& Kaiser, R. I. 2011, ApJ, 734, 78

Larson, R. B. 2003, Rep. Prog. Phys., 66, 1651

López-Sepulcre, A., Jaber, A. A., Mendoza, E., et al. 2015, MNRAS, 449, 2438

Lowenthal, M. S., Khanna, R. K., \& Moore, M. H. 2002, Spectrochim. Acta Part A, 58,73

Marcelino, N., Cernicharo, J., Tercero, B., et al. 2009, ApJ, 690, L27

Maret, S., Bergin, E. A., \& Lada, C. J. 2006, Nature, 442, 425

Mendoza, E., Lefloch, B., López-Sepulcre, A., et al. 2014, MNRAS, 445, 151

Mennella, V., Baratta, G. A., Esposito, A., et al. 2003, ApJ, 587, 727

Modica, P., \& Palumbo, M. E. 2010, A\&A, 519, A22
Modica, P., Palumbo, M. E., \& Strazzulla, G. 2012, Planet. Space Sci., 73, 425 Motiyenko, R. A., Tercero, B., Cernicharo, J., et al. 2012, A\&A, 548, A71

Mulas, G., Baratta, G. A., Palumbo, M. E., \& Strazzulla, G. 1998, A\&A, 333, 1025

Noble, J. A., Theule, P., Congiu, E., et al. 2015, A\&A, 576, A91

Nummelin, A., Bergman, P., Hjalmarson, A., et al. 2000, ApJS, 12, 213

Öberg, K. I., Boogert, A. C. A., Pontoppidan, K. M., et al. 2011, ApJ, 740, 109

Padovani, M., Galli, D., \& Glassgold, A. E. 2009, A\&A, 501, 619

Padovani, M., Hennebelle, P., \& Galli, D. 2013, A\&A, 560, A114

Paganini, L., DiSanti, M. A., Mumma, M. J., et al. 2014a, AJ, 147, 15

Paganini, L., Mumma, M. J., Villanueva, G. L., et al. 2014b, ApJ, 791, 122

Palumbo, M. E. 2006, A\&A, 453, 903

Palumbo, M. E., Leto, P., Siringo, C., \& Trigilio, C. 2008, ApJ, 685, 1033

Podio, L., Lefloch, B., Ceccarelli, C., et al. 2014, A\&A, 565, A64

Raunier, S., Chiavassa, T., Duvernay, F., et al. 2004, A\&A, 416, 165

Rodríguez-Fernández, N. J., Tafalla, M., Gueth, F., et al. 2010, A\&A, 516, A98

Rubin, R. H., Swenson, G. W., Jr, Benson, R. C., et al. 1971, ApJ, 169, L39

Rubin, M., Altwegg, K., Balsiger, H., et al. 2015, Science, 248, 232

Saladino, R., Crestini, C., Pino, S., et al. 2012, Phys. Life Rev., 9, 84

Saladino, R., Botta, G., Delfino, M., \& Di Mauro, E. 2013, Chem. Europ. J., 19, 16916

Schutte, W. A., Boogert, A. C. A., Tielens, A. G. G. M., et al. 1999, A\&A, 343, 966

Sicilia, D., Ioppolo, S., Vindigni, T., et al. 2012, A\&A, 543, A155

Soifer, B. T., Puetter, R. C., Russell, R. W., et al. 1979, ApJ, 232, L53

Strazzulla, G., \& Johnson, R. E. 1991, in Comets in the post-Halley Era, eds. R. L. Newburn, Jr., M. Neugebauer, \& J. Rahe (London: Kluwer, Publ. Co.), 243 Strazzulla, G., Baratta, G. A., Johnson, R. E., et al. 1991, Icarus, 91, 101

Strazzulla, G., Baratta, G. A., \& Palumbo, M. E. 2001, Spectrochim. Acta A, 57, 825

Yamada, H., \& Person, W. B. 1964, J. Chem. Phys., 41, 2478

Woods, P. M., Occhiogrosso, A., Viti, S., et al. 2015, MNRAS, 450, 1256

Ziegler, J. F., Biersack, J. P., \& Ziegler, M. D. 2008, The Stopping and Range of Ions in Solids (New York: Pergamon Press), 321 